\title{
Multiple Ratiometric Nanothermometry using Semiconductor BiFeO3 Nanowires and Quantitative Validation of Thermal Sensitivity
}

This paper was downloaded from TechRxiv (https://www.techrxiv.org).

\section{LICENSE}

CC BY 4.0

SUBMISSION DATE / POSTED DATE

$10-10-2021 / 14-10-2021$

\section{CITATION}

Kovur, Prashanthi; Kovur, Krishna M; Antic, Zeljka; Ahadi, Kaveh; Dramicanin, Miroslav D. (2021): Multiple Ratiometric Nanothermometry using Semiconductor BiFeO3 Nanowires and Quantitative Validation of Thermal Sensitivity. TechRxiv. Preprint. https://doi.org/10.36227/techrxiv.16780459.v1

$\mathrm{DOI}$ 


\title{
Multiple Ratiometric Nanothermometry using Semiconductor $\mathrm{BiFeO}_{3} \mathrm{Nanowires}$ and Quantitative Validation of Thermal Sensitivity
}

\author{
K. Prashanthi ${ }^{1}$, Krishna Mohan $K^{1}$, Željka Antici, ${ }^{1,2}$, Kaveh Ahadi ${ }^{1,3}$, Miroslav D. Dramicanin ${ }^{2}$, \\ ${ }^{1}$ University of Alberta, Edmonton, T6G 2V4, Canada \\ ${ }^{2}$ University of Belgrade, P.O.Box 522, 11001 Belgrade, Serbia \\ ${ }^{3}$ University of California, Santa Barbara, California 93106-5050, USA.
}

Abstract-Here, we report a very sensitive, non-contact, ratio-metric, and robust temperature sensing using a combination of conventional and negative thermal quenching (NTQ) mechanisms of semiconductor $\mathrm{BiFeO}_{3}$ (BFO) nanowires. Using this approach, we have demonstrated the absolute thermal sensitivity of $\sim 10 \times 10^{-3} \mathbf{K}^{-1}$ over the $300 \mathrm{~K}-438 \mathrm{~K}$ temperature range and the relative sensitivity of $0.75 \% \mathrm{~K}^{-1}$ at $300 \mathrm{~K}$. Further, we have validated thermal sensitivity of BFO nanowires quantitatively using linear regression and analytical hierarchy process (AHP) and found close match with the experimental results. These results indicated that BFO nanowires are excellent candidates for developing high-performance luminescence based temperature sensors.

Index Terms- Temperature sensors, Thermal quenching, Nanowires, Sensitivity validation

\section{INTRODUCTION}

Detection of temperature using changes in optical emission from nanomaterials is important in many fields such as biochemical processes, physiological processes, medical diagnostics and electronic devices ${ }^{1}$. The changes of luminescence properties of nanomaterials with temperature such as changes of absolute and relative emission intensities, lifetime values of excited states, emission rise times, peak positions, and emission bandwidths allow them to act as sensitive thermometers. Optical thermometry enables local temperature measurement of a system with sub-micron spatial resolution ${ }^{2-4}$. Temperature sensing through photoluminescence (PL) is based on the use of either nanoparticles or luminescent molecular probes as it offers a convenient 
optical measurement method ${ }^{1,5,6}$. Recently, the luminescence ratiometric nanothermometry performed via relative emission intensity measurements or luminescence intensity ratios gained a lot of attention because of its self-referencing capability, high precision, and high reliability ${ }^{7-9}$. The ratiometric nanothermometry method is unaffected by the variations in the measurement conditions and changes in the excitation source and can be potentially used in molecular nano thermometers or bioprobes ${ }^{10-14}$.

The PL quenching as a function of temperature is strongly associated with the energy difference between transition states and offers high sensitivity in temperature sensing ${ }^{15-17}$. Previous studies on ratiometric thermometry was mainly focussed on conventional thermal quenching of semiconductor materials or other nanophosphors where the emission intensities decrease with increase in tempearture ${ }^{18-20}$. Such PL quenching mechanism in semiconductors is mainly attributed to the delocalization of charge carriers and consequent trapping by non-radiative centers or energy states (with increasing temperature) which would result in decrease in the number of the charge carriers transiting from the final energy levels to the initial energy levels. Unlike the conventional thermal quenching in most semiconductors, the negative thermal quenching (NTQ) is the phenomenon where the number of charge carriers transitioning between final and initial energy states increase with increase in temperature. This NTQ behaviour in nanoscale semiconductors is mainly due to the characteristic surface states caused by lattice defects which are responsible for releasing trapped charge carriers in the recombination process at high temperatures. In our recent work, we have demonstrated that the semiconductor BFO nanowires show a rare phenomenon of NTQ of emission ${ }^{21}$, where emission intensity of certain peaks as a function of temperature shows an increase in luminescence intensity instead of generally observed trend of decrease in intensity. In continuation of our published work ${ }^{21}$, here, we report on a new way of monitoring temperature variations via NTQ mechanism of BFO nanowires. The multiple ratiometric temperature sensing using a combination of conventional and unconventional (NTQ effect) mechanism offers a promising strategy towards ultrahigh relative sensitivity probing of temperature.

\section{EXPERIMENTAL PROCEDURE}

The preparation, structural, optical, and electrical properties characterization of semiconductor BFO nanowires were published by us previously ${ }^{22-24}$. The temperature sensing via luminescence 
thermometry was performed using a Fluorolog-3 Model FL3-221 spectrofluorometer system (Horiba-Jobin-Yvon) over the temperature range $300 \mathrm{~K}$ to $438 \mathrm{~K}$. For temperature dependent PL studies, the BFO nanowires were deposited both on $\mathrm{Pt}$ coated $\mathrm{Si}\left(\mathrm{Si} / \mathrm{SiO}_{2} / \mathrm{Ti} / \mathrm{Pt}\right)$ and quartz substrates. The PL measurements were performed under continuous excitation from a $450 \mathrm{~W}$ xenon lamp at a wavelength of $320 \mathrm{~nm}(3.87 \mathrm{eV})$. The BFO nanowire samples were placed on a custom-made temperature controlled platform, and emission spectra were collected via an optical fiber bundle. The temperature of the BFO nanowire sample was controlled by a temperature control system (within the accuracy of $\pm 0.5 \quad \mathrm{~K}$ ) employing PID (proportional-integral-derivative) feedback loop equipped with a T-type thermocouple for temperature monitoring. The schematic of the experimental setup used for optical (luminescence) thermometry is shown in Fig. 1. The topography imaging of BFO nanowires on quartz substrate was done using atomic force microscopy (AFM) (Bruker Dimension Icon, USA). The ex-situ ellipsometric spectra $(\Psi, \Delta)$ were collected using a J.A. Woollam M2000DI at $70^{\circ}$ angle of incidence under $400 \mathrm{mTorr}$ vacuum at $325 \mathrm{~K}, 375 \mathrm{~K}, 425 \mathrm{~K}, 375 \mathrm{~K}, 525 \mathrm{~K}$, and $575 \mathrm{~K}$.

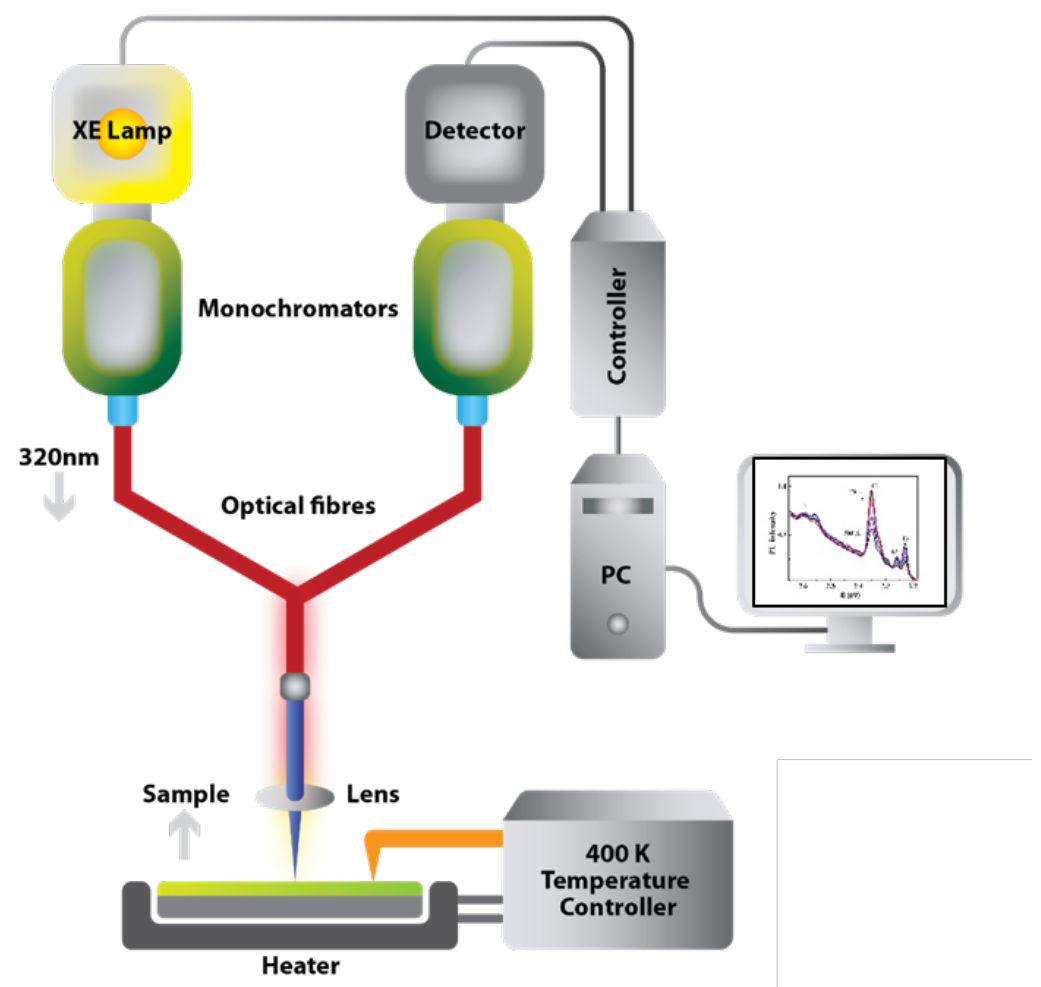

Fig. 1 Schematic of experimental setup used for optical thermometry 


\section{RESULTS AND DISCUSSIONS}

\section{A. Temperature dependent optical response}

Fig. 2(a) presents PL spectra of BFO nanowires for E2 defect emission measured in the temperature range of 300-438 K. The PL emission intensity for E2 emission increases with temperatures. Fig. 2(b) shows the evolution of temperature-dependent PL intensity of BFO nanowires for all 4 emissions (E1, E2, E3, and E4) measured at temperatures in the range of 300 $\mathrm{K}-438 \mathrm{~K}$. The four emission bands with maxima at $2.55 \mathrm{eV}(\mathrm{E} 1), 2.35 \mathrm{eV}$ (E2), $2.27 \mathrm{eV}$ (E3) and $2.23 \mathrm{eV}$ (E4) correspond to the band edge/near band emission (NBE) and defect level/surface state emission (DLE) as reported in our recent work ${ }^{21}$. As we can see from Fig. 2(b) that the PL intensity decreases for E1, E3, and E4 emissions, however, PL intensity increases for E2 emission. Since BFO nanowires provide multiple emission peaks, a ratiometric approach to luminescence thermometry could be used. Such ratiometric measurements are insensitive to fluctuations of excitation source light or other changes in measurement conditions and, therefore, act as self-referencing (i.e., measurements do not have to be referenced with any temperature standard $)^{2,25}$. When BFO nanowires are excited with UV light, the photons cause electrons to move into allowed excited states. However, when these electrons return to their equilibrium states, the excess energy is released as photon emission via radiative transition. In our previous work, we have reported that the presence of excess surface states in the band gap of BFO nanowires play an intermediate role during the emission process by capturing the excited carriers

and acting as radiation centers with enhanced emission ${ }^{21}$. The increase in temperature results in the release carriers from these surface states and when these carriers again recombine radiatively, they contribute to the green emission. 

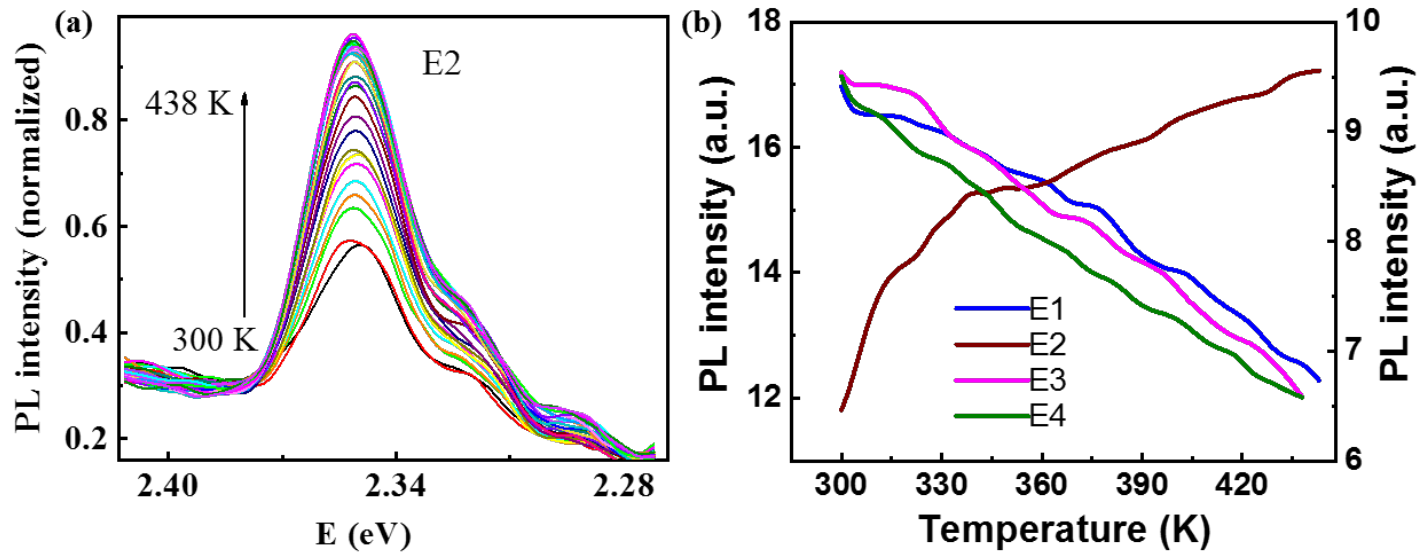

Fig. 2 (a) Temperature-dependent PL spectra of BFO nanowires for E2 emission. (b) Intensity of PL spectra plotted as a function of temperature for E1, E2, E3, and E4 emissions. Luminescence intensity increases with an increase in temperature for $2.35 \mathrm{eV}(\mathrm{E} 2)$ emission, whereas a decrease in PL intensity with temperature is observed for other emissions (E1, E3 and E4).

Fig. 3(a) presents optical images of BFO nanowire samples under the sunlight $(300 \mathrm{~K})$ and under UV $\left(\lambda_{\text {lamp }}=254 \mathrm{~nm}\right)$ illumination $(300 \mathrm{~K}, 330 \mathrm{~K}$, and $360 \mathrm{~K})$. From the optical images, it can be observed that the BFO nanowires emit green luminescence in the presence of UV light and the intensity of green luminescence increases as the temperature of the sample is increased from 300 K to $360 \mathrm{~K}$. Fig. 3(b) demonstrates an optical transparency of BFO nanowires (deposited on the quartz substrate) under sunlight and under UV illumination at $300 \mathrm{~K}$. Our Institutions' logo is clearly seen under the ambient sunlight as well as under UV light demonstrating BFO nanowires have good transparency. Moreover, under UV light excitation, beside good transparency, the green photoluminescence from nanowires is clearly visible. The enlarged portion of Fig. 3(b) shows an AFM image of BFO nanowires. As demonstrated by the AFM topography, high density of nanowires was achieved on the quartz substrate. Generally, the nanowires have a diameter distribution of $90-250 \mathrm{~nm}$ with an average length of tens of micrometers. Further, we have investigated the temperature dependent refractive index $(\mathrm{n})$ and extinction coefficient $(\mathrm{k})$ of BFO nanowires using ellipsometry. The ellipsometric spectra of BFO nanowires were evaluated using the Cauchy model for the quartz substrates. A three-medium optical model consisting substrate/bulk film/ambient was employed. The optical response of the BFO nanowires is described by a Tauc-Lorentz oscillator in the band gap energy region ${ }^{26,27}$. The Bruggeman Effective Medium Approximation (EMA) was employed to count for the voids between the BFO 
nanowires. Eventually, substrate back side correction was included. Parameters of Tauc-Lorentz oscillators were fitted with least squares regression analysis leading to low mean square error $(<10)$. Fig. 3(c) reveals the Psi and Delta with an aforementioned optical model for BFO nanowires. Parameters of Tauc-Lorentz oscillators were fitted with least squares regression analysis leading to low mean square error $(<10)$. Fig. 3(d) shows the extracted refractive index (n) and extinction coefficient (k) for BFO nanowires, accordingly. Extracted refractive index (n) values agree with ones found in the literature ${ }^{28}$. The sub-bandgap extinction coefficient $(\mathrm{k})$ for BFO nanowires can be due to the contribution from surface states. Both the extracted refractive index (n) and extinction coefficient (k) seem to be insensitive to temperature (Fig. 3(f)). Furthermore, band gap also qualitatively does not show a drastic change with temperature in this range.

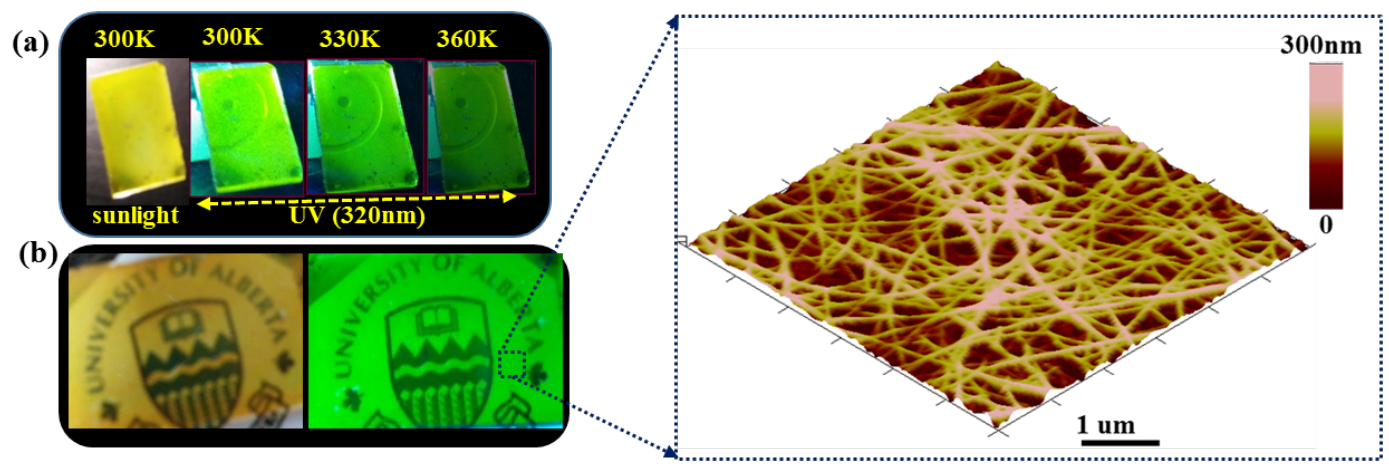

(c)

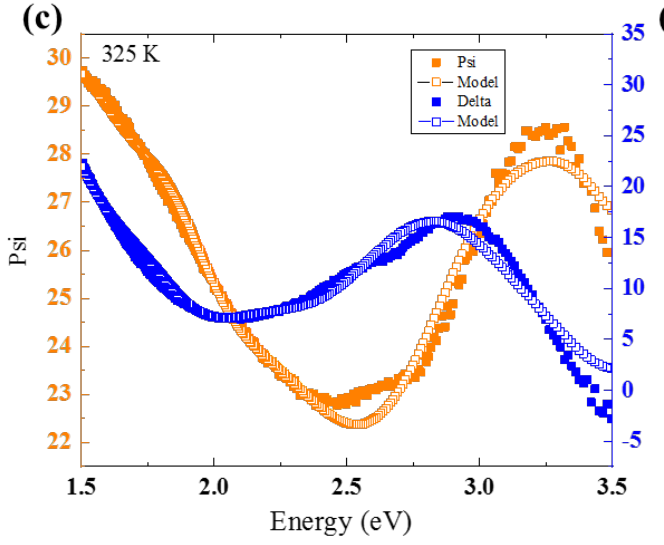

(d)

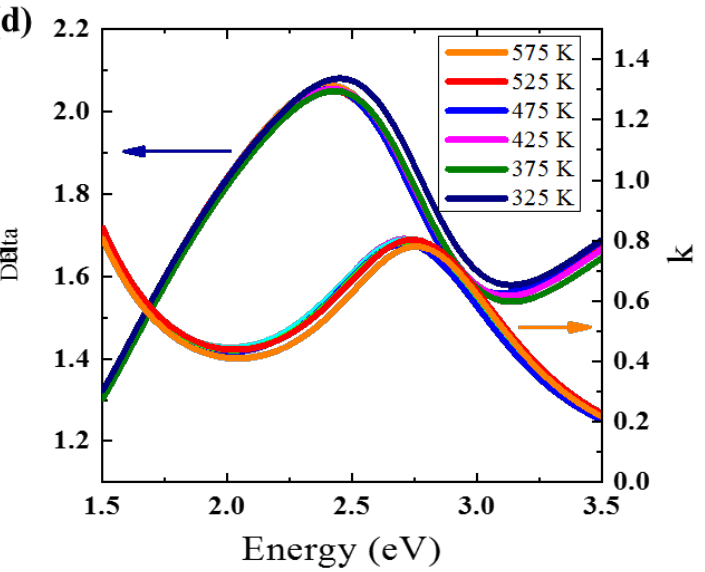




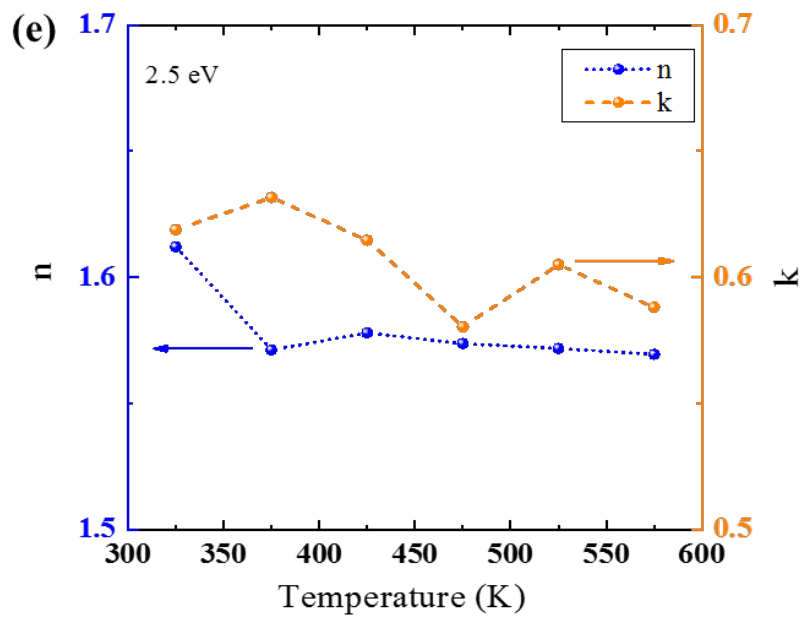

Fig 3 (a) Optical images of BFO nanowire samples under the sunlight (300 K) and under UV $\left(\lambda_{\text {lamp }}=254 \mathrm{~nm}\right)$ illumination $(300 \mathrm{~K}, 330 \mathrm{~K}$, and $360 \mathrm{~K})(\mathrm{b})$ Optical transparency of BFO nanowires under sunlight and under UV illumination $\left(\lambda_{\text {lamp }}=254 \mathrm{~nm}\right)$ at $300 \mathrm{~K}$. Enlarged image shows an AFM image of BFO nanowires (c) The raw Psi and Delta measured by ellipsometry and fitted optical model. The real and imaginary component of refractive index with respect to (d) incident photon energy $(325 \mathrm{~K}, 375 \mathrm{~K}, 425 \mathrm{~K}, 375 \mathrm{~K}, 525 \mathrm{~K}$, and $575 \mathrm{~K}$ ) and (e) temperature (at $2.5 \mathrm{eV}$ ).

\section{B. Temperature dependent luminescence decay lifetime}

The calculated lifetime values of BFO nanowires as a function of temperature for E2 emission is presented in Fig. 4. As observed from temperature dependant luminescence lifetime studies, the decay time is $\sim 160$ us. Such high decay time is expected because of the fact that the transitions from surface states are forbidden, which results in slow emission rate. Further, it can be noted from Fig. 5 that the lifetime is independent of the temperature change, resembling the static quenching behavior and can be explained in terms of multiple trapping and de-trapping process as explained in our earlier work ${ }^{21}$. 


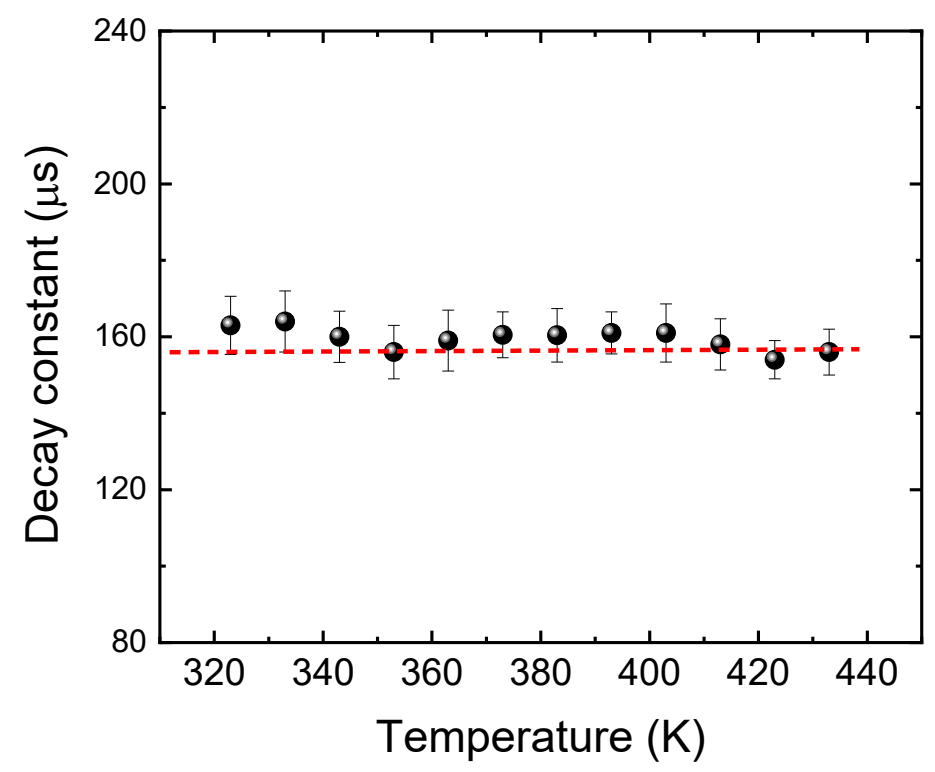

Fig. 4 Lifetime values of BFO nanowires obtained for E2 emission as a function of temperature

\section{Thermal sensitivity analysis}

Fig. 5(a) shows a plot of the temperature dependence of the luminescence intensity ratio (LIR) obtained as ratios of E2 emission with other three (E1, E3 and E4). In principle, for the ratiometric sensing, no knowledge regarding the physics underlying the temperature quenching is needed. It is sufficient to approximate the experimental data for further use as a calibration curve $^{29}$. For the case of BFO nanowires, LIR data are fitted with the linear function as shown in Fig. 5(b), and fitting parameters are given in the Table 1. The most important Figures of merits for luminescence thermometry are the absolute sensor sensitivity, $S_{a}$ defined as the rate at which LIR changes with the temperature, and the relative sensor sensitivity, $S_{r}$ defined as the normalized absolute sensor sensitivity with respect to the measured value: ${ }^{2}$

$$
\begin{aligned}
& \mathrm{S}_{\mathrm{a}}=\frac{|\partial \mathrm{LIR}|}{\partial \mathrm{T}} \\
& S_{r}=100 \% \times\left|\frac{1}{L I R} \frac{\partial L I R}{\partial T}\right|
\end{aligned}
$$

The absolute and relative sensitivities of BFO nanowires calculated from Equation $1 \& 2$ are plotted in Fig. 5(b), and are listed in Table 1. Relative sensitivities achieved with BFO nanowires are similar (slightly lower) but with larger measurement ranges than those reported on 
ratiometric luminescence thermometry with other semiconductors. Relative sensitivities of 0.9 and $1.3 \% \mathrm{~K}^{-1}$ have been found for differently sized nanoparticles of $\mathrm{Zn}_{0.99} \mathrm{Mn}_{0.01} \mathrm{Se} / \mathrm{ZnCdSe}$ in the temperature range of $293 \mathrm{~K}-373 \mathrm{~K}^{30}$, and $0.9 \% \mathrm{~K}^{-1}$ with $\mathrm{CdSe} / \mathrm{ZnSe}$ and $\mathrm{CdTe} / \mathrm{ZnS}$ over the narrow temperature range of $293 \mathrm{~K}-320 \mathrm{~K}^{31}$. It should be note that $\mathrm{Zn}_{0.99} \mathrm{Mn}_{0.01} \mathrm{Se} / \mathrm{ZnCdSe}$ luminescence thermometry has been realized for emission from both semiconductors and $\mathrm{Mn}^{2+2}$.

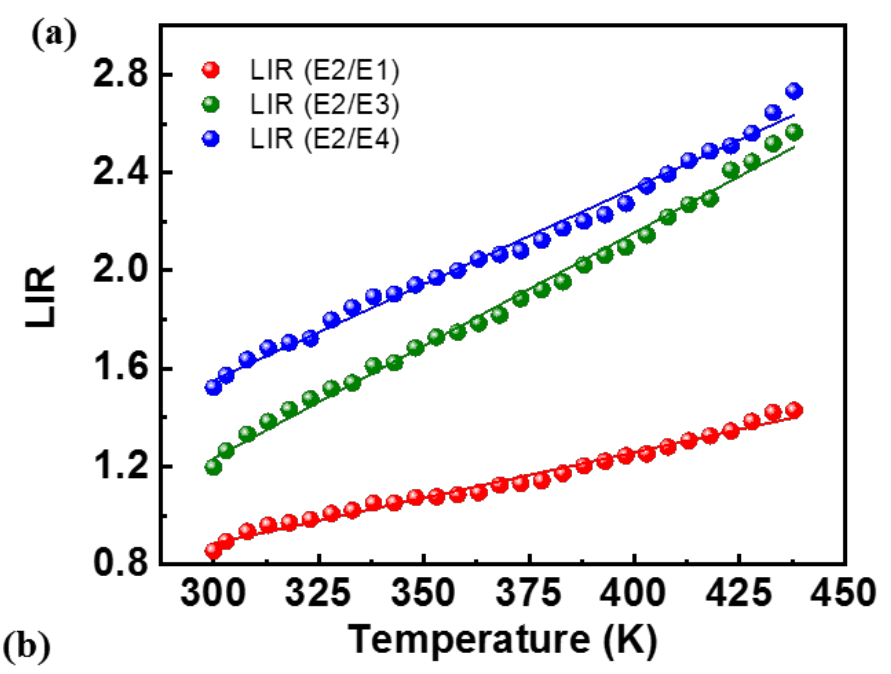

(b)

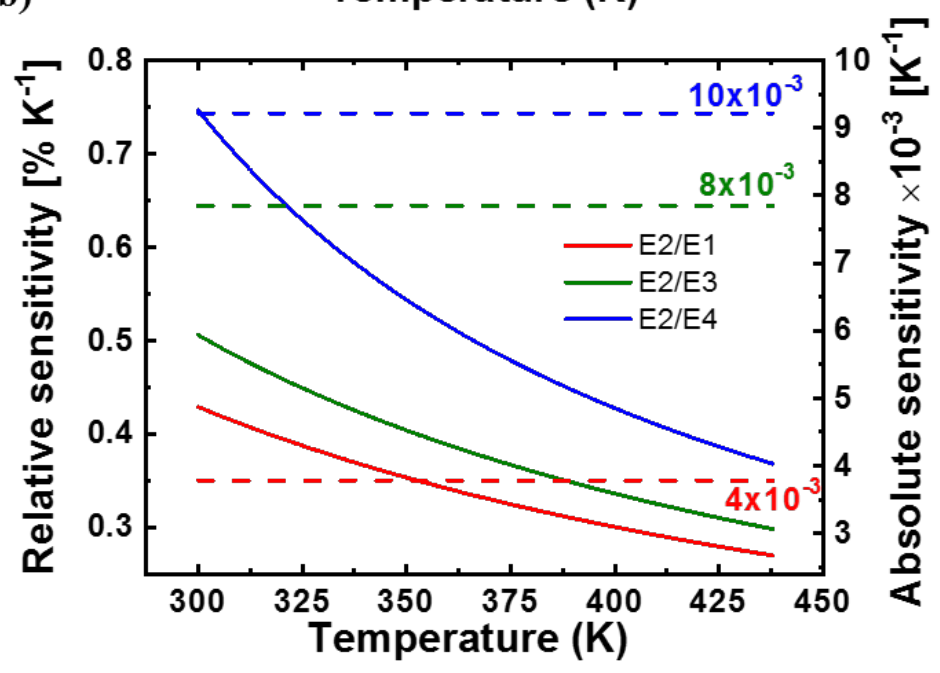

Fig. 5 (a) Luminescence intensity ratio (LIR) values of BFO nanowires as a function of temperature (circles). LIR values were obtained as the ratio between peak intensities for E1, E3, and E4 with respect to E2. (b) Relative and absolute sensor sensitivity obtained from LIR measurements as a function of temperature $(300 \mathrm{~K}-438 \mathrm{~K})$.

\begin{tabular}{lllll}
\hline LIR & Temperature & Temperature & \multicolumn{2}{c}{ Thermal sensitivity } \\
ratio & range & dependence & $S_{a}\left(\mathbf{K}^{-1}\right)$ & $S_{r \max }\left(\% \mathbf{K}^{-1}\right)$ \\
& & & (a 300K \\
\hline
\end{tabular}




\begin{tabular}{lllll}
\hline E2/E1 & $300 \mathrm{~K}-438 \mathrm{~K}$ & $-0.25+0.00378 \cdot T$ & $4 \times 10^{-3}$ & 0.43 \\
E2/E3 & $300 \mathrm{~K}-438 \mathrm{~K}$ & $-0.25+0.00785 \cdot T$ & $8 \times 10^{-3}$ & 0.51 \\
E2/E4 & $300 \mathrm{~K}-438 \mathrm{~K}$ & $-1.53+0.00922 \cdot T$ & $10 \times 10^{-3}$ & 0.75
\end{tabular}

Table 1: Table summarizing figure of merits of the luminescence thermometry using negative quenching effect of E2 (2.35 eV) emission of BFO nanowires.

\section{Validation of thermal sensitivity using Analytical Hierarchy Process (AHP)}

Based on the data obtained from E2/E4 emission, we have developed intercept equations in $y=a+b x$ form, where $y$ represents dependent variable (relative emission intensity) and $x$ is an independent variable (temperature) through simple linear regression and log value regression. Equation 3 represents linear regression with log value and equation 4 represents linear regression with respective evaluated $\mathrm{R}^{2}$ values. The curve fitting plot and as well equations (with and without $\log$ value) with $\mathrm{R}^{2}$ values are represented in Fig. 6.

$$
\begin{aligned}
& y=-1.313 \ln (x)+8.22 ; R^{2}=0.9961 . . \\
& y=-0.004 x+1.94 ; R^{2}=0.9933 . .
\end{aligned}
$$

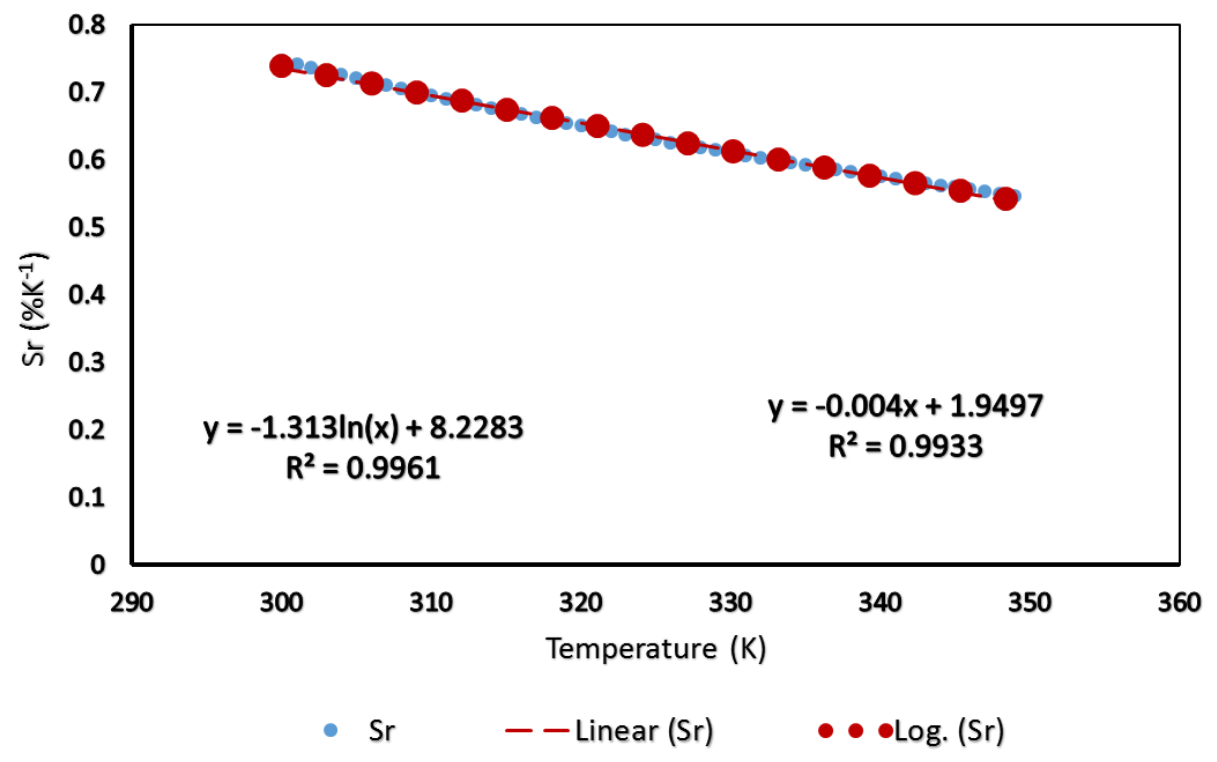

Fig. 6 Prediction of relative sensitivity $\left(\mathrm{S}_{\mathrm{r}}\right)$ for E2/E4 as a function of temperature $(300 \mathrm{~K}-438$ K) using linear regression.

One of the evaluated parameters will help the expert instantly with linear regression is $\mathrm{R}^{2}$. The statistical significance of $\mathrm{R}^{2}$ is a degree of how well our considered application is appropriately 
fitting the actual data close to linearity relationship. The linearity relationship in this case is between temperature and E2/E4 emission intensity. The value of $\mathrm{R}^{2}$ should always lies between the spectrum of 0 to 1 . If the value is close to 1 , then the observed variance of linearity is high and whereas if it is close to 0 then the linear relationship is considered to be weak or low. However, we can predict the target signal in either of the cases with a high or low linearity relationships. In our case the $\mathrm{R}^{2}$ (log - linear) is calculated to be 0.9961 while in simple linear as 0.9933 (without $\log$ ). Since, the $\mathrm{R}^{2}$ value is close to 1 , we conclude the relationship of sensitivity with temperature is strongly linear. Since, the regression results are found linear, we have further conducted validation of thermal sensitivity of BFO nanowires using LIR ratios at different temperatures via the Analytical Hierarchy Process (AHP) model ${ }^{32-36}$. The validation model analysis of thermal sensitivity has been accomplished using AHP quantitatively. The AHP, a prominent methodology founded on multi-criteria decision-making model established by Saaty ${ }^{33}$ aims at quantifying influencing factors. In our paper, we have quantified emission intensities at different temperatures, in other words at varying levels of abstraction of temperature sensing using semiconductor BFO nanowires and further observed its sensitive analysis. The quantification is performed through the data obtain from LIR ratios (E2/E1, E2/E3, E2/E4) through a series of experiments. Thus, we translate the acquired data patterns into the scale 1 to 9 (based on the emission intensity) of the AHP model suggested by Saaty ${ }^{32}$. To recall, here, we present a brief classification of steps involved in AHP quantitative evaluation through series of steps: (a) defining the objective of the considered application problem, (b) modeling influencing factors into hierarchy at various appropriate levels, (c) forming pair-wise comparison matrices among the factors at each level of the hierarchy, (d) evaluation of the matrices through weigh vectors via principal Eigen vector ${ }^{33}$, (e) pooling of all assessed weigh vectors according to the hierarchy model, and lastly (f) final quantification evaluation and sensitivity analysis ${ }^{37}$.

Specifically, in this paper, we have investigated the merits of AHP for quantitative validation of the thermal sensitivity of BFO nanowires and performed the following assessments:

- quantified and validated thermal sensitivity using LIR ratios (E2/E1, E2/E3, E2/E4) at $300 \mathrm{~K}$

- $\quad$ similarly, further quantified LIR ratios at $346 \mathrm{~K}$ and $440 \mathrm{~K}$ 
- finally, node sensitivities at each of the LIR ratios w.r.t overall temperature range of 300 $\mathrm{K}-440 \mathrm{~K}$

Thus, we applied steps (a to f) AHP algorithm mentioned above to quantify thermal sensitivity of BFO nanowires using LIR ratios. The first step (a) been defining the objective - the validation of thermal sensitivity of LIR ratios quantitatively. In step (b), based on the experimental results for thermal sensitivity of BFO nanowires using LIR ratios at different temperature, we have modelled in a 2 layered hierarchy, shown Fig. 7. The first level consists of considered three temperatures $\mathrm{T} 1(300 \mathrm{~K}), \mathrm{T} 2(346 \mathrm{~K})$, and $\mathrm{T} 3(440 \mathrm{~K})$. The second layer and the last level includes LIR ratio (E2/E1, E2/E3, E2/E4).

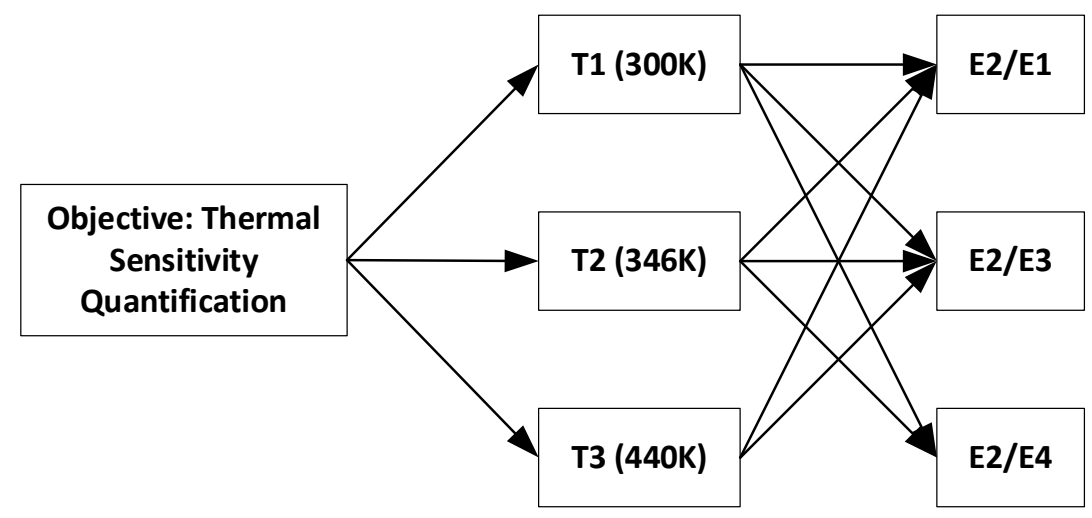

Fig. 7 AHP model for validation of thermal sensitivity

In the steps (c), formulated pair-wise comparison matrices with one pair matrix at the first level and three pair-wise matrices at the second level. Next in the step (d), assessment of four matrices through weigh vectors evaluations has been performed. The formulation and evaluations of matrices from steps (c) and (d) are shown in the Table 2. All the weight vectors calculated from four matrices in step (d) are pooled and organized via. in the form aggregation matrix shown in the Table 3, this completes the step (e). Finally, in the step (f), we perform concluding evaluation from the aggregation matrix (from step $\mathrm{d}$ ) through a customized equation to evaluate the overall node sensitivities (at each of the LIR ratios) at the overall temperature range of $300 \mathrm{~K}-440 \mathrm{~K}$ of the considered application shown in Table 4.

Based on AHP modelling and assessment, we have validated the following pieces of quantitative analysis: (i) Table 2 (a) validated thermal sensitivity using LIR ratios of E2/E1, E2/E3, E2/E4 at $300 \mathrm{~K}$ (room temperature); (ii) Similarly, further quantified LIR ratio at $346 \mathrm{~K}$ and $440 \mathrm{~K}$, shown in the Table 2 (b); and 2 (c); and (iii) Fig. 8 demonstrates sensitivity analysis through clusters 
LIR ratios at $300 \mathrm{~K}, 346 \mathrm{~K}$, and $440 \mathrm{~K}$ and finally, Fig. 9 depicts the node sensitivities at $\mathrm{p}=0.5$ (at each of the LIR ratios) at the overall temperature range of $300 \mathrm{~K}-440 \mathrm{~K}$.

Table 2 Formulation of Pair-wise matrices and assessments Level 1

\begin{tabular}{|c|c|c|c|c|}
\hline & T1 & T2 & T3 & $\begin{array}{c}\text { Weigh } \\
\text { vector }\end{array}$ \\
\hline T1 & 1 & $1 / 5$ & $1 / 6$ & 0.0811 \\
\hline T2 & 5 & 1 & $1 / 2$ & 0.3420 \\
\hline T3 & 6 & 2 & 1 & 0.5769 \\
\hline
\end{tabular}

(a) Relative importance among T1, T2, \&T3 Level 2

\begin{tabular}{|c|c|c|c|c|}
\hline T1 & E2/E1 & E2/E3 & E2/E4 & $\begin{array}{c}\text { Weigh } \\
\text { vector }\end{array}$ \\
\hline E2/E1 & 1 & 1 & $1 / 3$ & $\mathbf{0 . 2 2 1 1}$ \\
\hline E2/E3 & 1 & 1 & 1 & $\mathbf{0 . 3 1 8 9}$ \\
\hline E2/E4 & 3 & 1 & 1 & $\mathbf{0 . 4 5 9 9}$ \\
\hline
\end{tabular}

(b) Variations of LIR (E2/E1, E2/E3,\& E2/E4) @ T1(300K)

\begin{tabular}{|c|c|c|c|c|}
\hline T2 & E2/E1 & E2/E3 & E2/E4 & $\begin{array}{c}\text { Weigh } \\
\text { vector }\end{array}$ \\
\hline E2/E1 & 1 & $1 / 3$ & $1 / 4$ & $\mathbf{0 . 1 2 1 9}$ \\
\hline E2/E3 & 3 & 1 & $1 / 2$ & $\mathbf{0 . 3 1 9 6}$ \\
\hline E2/E4 & 4 & 2 & 1 & $\mathbf{0 . 5 5 8 4}$ \\
\hline
\end{tabular}

(c) Variations of LIR (E2/E1, E2/E3,\& E2/E4) @ T2 (346K)

\begin{tabular}{|c|c|c|c|c|}
\hline T3 & E2/E1 & E2/E3 & E2/E4 & $\begin{array}{c}\text { Weigh } \\
\text { vector }\end{array}$ \\
\hline E2/E1 & 1 & $1 / 6$ & $1 / 7$ & $\mathbf{0 . 0 7 6 0}$ \\
\hline E2/E3 & 6 & 1 & 1 & $\mathbf{0 . 4 5 2 7}$ \\
\hline E2/E4 & 7 & 1 & 1 & $\mathbf{0 . 4 7 1 2}$ \\
\hline
\end{tabular}

(d) Variations of LIR (E2/E1, E2/E3,\& E2/E4)@ T3 (440K)

Table 3 Aggregation Matrix

\begin{tabular}{|c|c|c|c|c|}
\hline Temp $\rightarrow$ & $\begin{array}{c}\mathrm{T} 1 \\
(300 \mathrm{~K})\end{array}$ & $\begin{array}{c}\mathrm{T} 2 \\
(346 \mathrm{~K})\end{array}$ & $\begin{array}{c}\mathrm{T} 3 \\
(440 \mathrm{~K})\end{array}$ & Evaluation comments \\
\hline $\mathbf{A}_{\mathbf{i}} \rightarrow$ & $\begin{array}{c}0.0811 \\
\left(a_{1}\right)\end{array}$ & $\begin{array}{c}0.3420 \\
\left(\mathrm{a}_{2}\right)\end{array}$ & $\begin{array}{c}0.5769 \\
\left(a_{3}\right)\end{array}$ & $\begin{array}{l}\text { Relative importance among } \mathrm{T} 1, \mathrm{~T} 2 \text {, } \\
\& \mathrm{~T} 3\end{array}$ \\
\hline LIR Ratios $\downarrow$ & \multicolumn{3}{|c|}{$\mathbf{A}_{\mathbf{i j} \downarrow} \downarrow$} & \\
\hline E2/E1 & $\begin{array}{c}0.2211 \\
\left(\mathrm{a}_{11}\right)\end{array}$ & $\begin{array}{c}0.1219 \\
\left(\mathrm{a}_{21}\right)\end{array}$ & $\begin{array}{c}0.0760 \\
\left(\mathrm{a}_{31}\right)\end{array}$ & \multirow[t]{3}{*}{$\begin{array}{l}\text { Variations of LIR (E2/E1, E2/E3,\& } \\
\text { E2/E4) w.r.t to T1, T2, \& T3 }\end{array}$} \\
\hline E2/E3 & $\begin{array}{c}0.3189 \\
\left(\mathrm{a}_{12}\right) \\
\end{array}$ & $\begin{array}{c}0.3196 \\
\left(\mathrm{a}_{22}\right)\end{array}$ & $\begin{array}{c}0.4527 \\
\left(\mathrm{a}_{32}\right)\end{array}$ & \\
\hline E2/E4 & $\begin{array}{c}0.4599 \\
\left(a_{13}\right)\end{array}$ & $\begin{array}{c}0.5584 \\
\left(a_{23}\right)\end{array}$ & $\begin{array}{c}0.4712 \\
\left(\mathrm{a}_{33}\right)\end{array}$ & \\
\hline
\end{tabular}


Table 4 Final evaluation

Let us denote $\mathrm{A}^{(1)}=\mathrm{a}_{1}, \mathrm{~A}^{(2)}=\mathrm{a}_{2}, \mathrm{~A}^{(3)}=\mathrm{a}_{3}$

Overall thermal sensitivity of LIR ratio (E2/E1, E2/E3, \& E2/E4) over the temperature range of $300 \mathrm{~K}-440 \mathrm{~K}$

$=\sum_{k=1}^{3}\left[\sum_{i=1}^{i_{k}} A_{i}^{(k)} A_{i j}^{(k)}\right] ;$ for $\mathrm{j}=1,2.3 ; \mathrm{i}_{1}=3$ (in our case)

$=0.1009(\mathrm{E} 2 / \mathrm{E} 1)$

$0.3960(E 2 / E 3)$

$0.5029(E 2 / E 4)$

The ultimate aim of validation of the AHP results is to compare the experimental trend of thermal sensitivity of semiconductor BFO nanowires using LIR ratios. For illustrative purpose, we have compared the results of AHP for LIR ratios of E2/E1, E2/E3, E2/E4) at 300K with the results of Table 1 and Fig. 5 (a). We found a close match between AHP model with the simulation result (Table 1) and experimental tendency pattern results in Fig 5 (a). The comparison of results obtained from these methods are shown in Table 5. Therefore, our analytical validation results endorse the experimental analysis results of thermal sensitivity of semiconductor BFO nanowires.

Table 5 Thermal sensitivity validations

\begin{tabular}{|c|c|c|c|}
\hline & \multicolumn{2}{|c|}{$\begin{array}{c}S_{r} \max \left(\% \mathbf{K}^{-1}\right) @ 30 \mathrm{30K} \\
(\text { Table 1) }\end{array}$} & $\begin{array}{c}\text { AHP model } \\
\text { (Table 2(b)) }\end{array}$ \\
\hline LR values & Ideal values & Normalized & \multicolumn{1}{c|}{ Normalized } \\
\hline E2/E1 & $\mathbf{0 . 4 3}$ & $\mathbf{0 . 2 5 4}$ & $\mathbf{0 . 2 2 1 1}$ \\
\hline E2/E3 & $\mathbf{0 . 5 1}$ & $\mathbf{0 . 3 0 1}$ & $\mathbf{0 . 3 1 8 9}$ \\
\hline E2/E4 & $\mathbf{0 . 7 5}$ & $\mathbf{0 . 4 4 3}$ & $\mathbf{0 . 4 5 9 9}$ \\
\hline
\end{tabular}




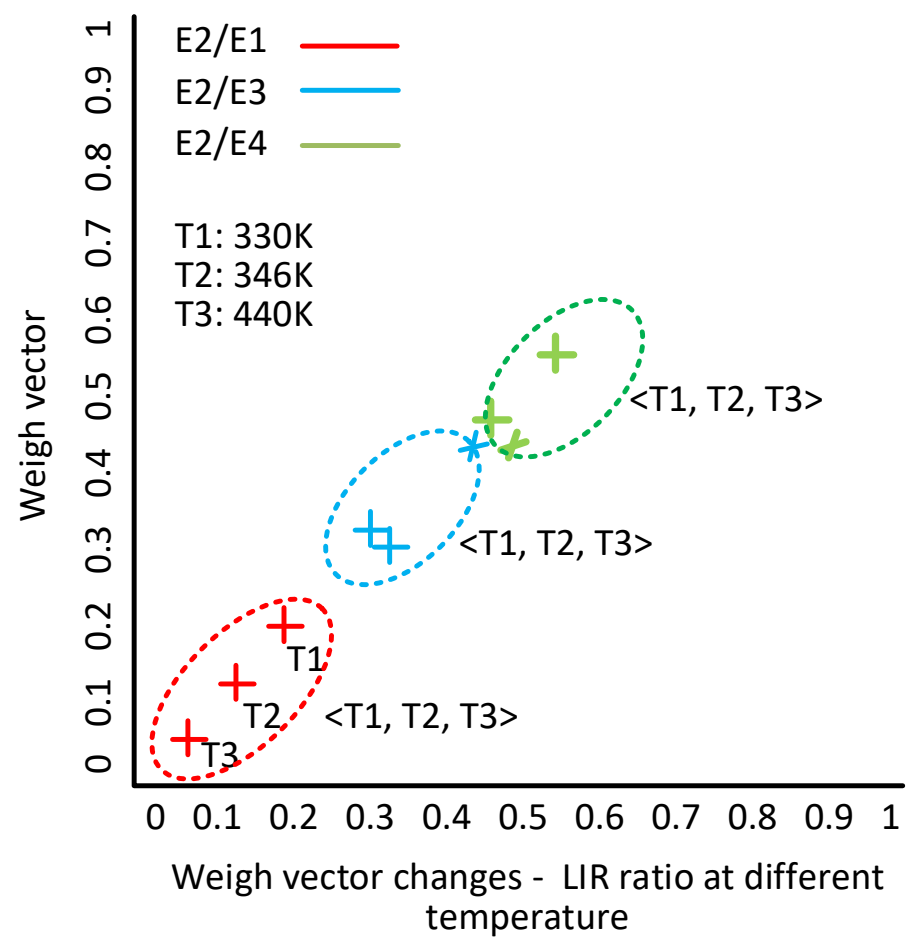

Fig. 8 Sensitivity analysis through clusters LIR ratios at 300K, 346K, and 440K

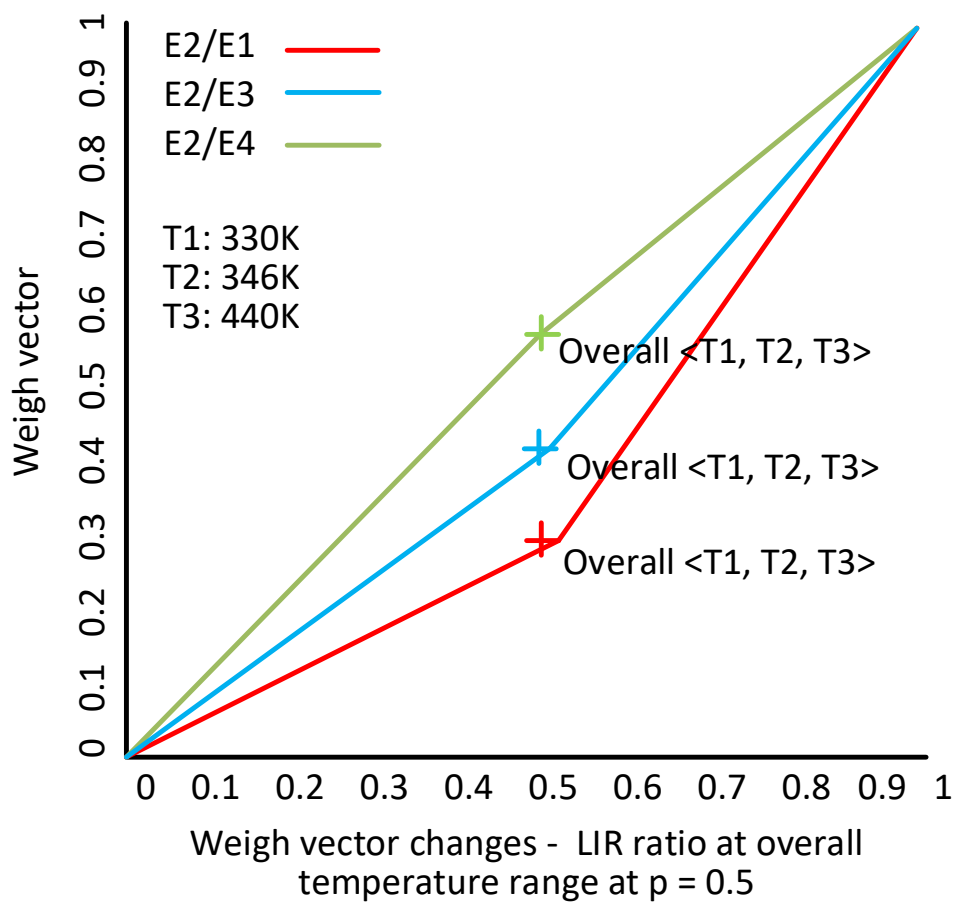

Fig. 9 Node sensitivities of LIR ratios $(p=0.5)$ at the overall temperature range $(300 \mathrm{~K}-440 \mathrm{~K})$ 


\section{CONCLUSION}

Multiple peaks in the emission spectrum of BFO nanowires enabled the use of ratio-metric luminescence temperature sensing in the range of $300 \mathrm{~K}-438 \mathrm{~K}$ with the relative sensitivity as high as $0.75 \% \mathrm{~K}^{-1}$. Additionally, using a linear regression and AHP, the quantitative validation has been performed for thermal sensitivity of BFO nanowires using LIR ratios at three different temperatures $(300 \mathrm{~K}, 346 \mathrm{~K}$, and $440 \mathrm{~K})$ and as well at overall temperature range of $300 \mathrm{~K}-440$ $\mathrm{K}$. In order to demonstrate quantitative validation of the AHP model, we have compared LIR ratio $(\mathrm{E} 2 / \mathrm{E} 1)$ at room temperature $(300 \mathrm{~K})$ with the experimental results and found a close match. In conclusion, our studies indicate that BFO nanowires can serve as potential candidate for multiple ratiometric nanothermometry based temperature sensors.

\section{DECLARATION}

\section{Ethics approval and consent to participate}

Not applicable.

\section{Consent for publication}

Not applicable.

\section{Competing interests}

The author(s) declare(s) that they have no competing interests.

\section{Funding}

This work was supported by Canada Excellence Research Chairs (CERC) Program and the Serbian Ministry of Education, Science and Technological development (project number 17022).

\section{Authors' contributions}

All the authors contributed for the design of experiments, data analysis, and preparation of the manuscript. All authors read and approved the final manuscript.

\section{Acknowledgements}


Authors would like to thank Dr. Thomas Thundat for useful discussions.

\section{Availability of data and material}

All the data generated and analyzed during this study is included in this manuscript.

\section{REFERENCES}

(1) Wang, X.; Wolfbeis, O. S.; Meier, R. J. Luminescent Probes and Sensors for Temperature. Chem. Soc. Rev. 2013, 42 (19), 7834.

(2) Brites, C. D. S.; Lima, P. P.; Silva, N. J. O.; Millán, A.; Amaral, V. S.; Palacio, F.; Carlos, L. D. Thermometry at the Nanoscale. Nanoscale 2012, 4 (16), 4799.

(3) Vetrone, F.; Naccache, R.; Zamarro, A.; Juarranz de la Fuente, A.; Sanz-Rodríguez, F.; Martinez Maestro, L.; Martı Rodriguez, E.; Jaque, D.; Garcia Solé, J.; Capobianco, J. a. Temperature Sensing Using Fluorescent Nanothermometers. ACS Nano 2010, 4 (6), 3254-3258.

(4) Jaque, D.; Vetrone, F. Luminescence Nanothermometry. Nanoscale 2012, 4 (15), 4301.

(5) Garcia, D. J.; Sole, J. G. Thermometry at the Nanoscale: Techniques and Selected Applications. In RSC Nanoscience \& Nanotechnology No. 38; Carlos;, L. D., Palacio, F., Eds.; The Royal Society of Chemistry, 2016; pp 83-123.

(6) Wang, S.; Westcott, S.; Chen, W. Nanoparticle Luminescence Thermometry. J. Phys. Chem. B 2002, 106 (43), 11203-11209.

(7) Dramićanin, M. D.; Antić, Ž.; Ćulubrk, S.; Ahrenkiel, S. P.; Nedeljković, J. M. SelfReferenced Luminescence Thermometry with Sm3+doped TiO2 Nanoparticles. Nanotechnology 2014, 25 (48), 485501.

(8) Antić, Ž.; Dramićanin, M. D.; Prashanthi, K.; Jovanović, D.; Kuzman, S.; Thundat, T. Pulsed Laser Deposited Dysprosium-Doped Gadolinium-Vanadate Thin Films for Noncontact, Self-Referencing Luminescence Thermometry. Adv. Mater. 2016, 28 (35).

(9) Lojpur, V.; Ćulubrk, S.; Dramićanin, M. D. Ratiometric Luminescence Thermometry with Different Combinations of Emissions from Eu3+ Doped Gd2Ti2O7 Nanoparticles. $J$. Lumin. 2015, 1-5.

(10) Kamimura, M.; Matsumoto, T.; Suyari, S.; Umezawa, M.; Soga, K. Ratiometric NearInfrared Fluorescence Nanothermometry in the OTN-NIR (NIR II/III) Biological Window 
Based on Rare-Earth Doped $\beta-N a Y F 4$ Nanoparticles. J. Mater. Chem. B 2017, 5 (10), $1917-1925$.

(11) Dong, N.-N.; Pedroni, M.; Piccinelli, F.; Conti, G.; Sbarbati, A.; Ramírez-Hernández, J. E.; Maestro, L. M.; la Cruz, M. C.; Sanz-Rodriguez, F.; Juarranz, A.; et al. NIR-to-NIR Two-Photon Excited CaF2:Tm3+,Yb3+ Nanoparticles: Multifunctional Nanoprobes for Highly Penetrating Fluorescence Bio-Imaging. ACS Nano 2011, 5 (11), 8665-8671.

(12) Smith, A. M.; Mancini, M. C.; Nie, S. Bioimaging: Second Window for in Vivo Imaging. Nat. Nanotechnol. 2009, 4 (11), 710-711.

(13) Jaque, D.; Richard, C.; Viana, B.; Soga, K.; Liu, X.; Solé, J. G. Inorganic Nanoparticles for Optical Bioimaging. Adv. Opt. Photon. 2016, 8 (1), 1-103.

(14) Hemmer, E.; Benayas, A.; Légaré, F.; Vetrone, F. Exploiting the Biological Windows: Current Perspectives on Fluorescent Bioprobes Emitting above 1000 Nm. Nanoscale Horizons 2016, 1 (3), 168-184.

(15) Paje, S. E.; Llopis, J. Temperature Dependence of the Photoluminescence and Phosphorescence Time Decay of Magnesia-Stabilized Zirconia. Appl. Phys. A Solids Surfaces 1992, 55 (6), 523-528.

(16) Fang, Y.-C.; Chu, S.-Y.; Kao, P.-C.; Chuang, Y.-M.; Zeng, Z.-L. Energy Transfer and Thermal Quenching Behaviors of CaLa[Sub 2](MoO[Sub 4])[Sub 4]:Sm[Sup 3+],Eu[Sup 3+] Red Phosphors. J. Electrochem. Soc. 2011, 158 (2), J1.

(17) Đačanin, L. R.; Lukić-Petrović, S. R.; Petrović, D. M.; Nikolić, M. G.; Dramićanin, M. D. Temperature Quenching of Luminescence Emission in Eu3+- and Sm3+-Doped YNbO4 Powders. J. Lumin. 2014, 151, 82-87.

(18) Antić, Ž.; Dramićanin, M. D.; Prashanthi, K.; Jovanović, D.; Kuzman, S.; Thundat, T. Pulsed Laser Deposited Dysprosium-Doped Gadolinium - Vanadate Thin Films for Noncontact, Self-Referencing Luminescence Thermometry. Adv. Mater. 2016, 28 (35), $7745-7752$.

(19) Lojpur, V.; Nikolić, M. G.; Jovanović, D.; Medić, M.; Antić, Ž.; Dramićanin, M. D. Luminescence Thermometry with Zn2SiO4:Mn2 Powder. Appl. Phys. Lett. 2013, 103 (14), 2013-2016.

(20) Yu, H.; Su, W.; Chen, L.; Deng, D.; Xu, S. Excellent Temperature Sensing Characteristics of Europium Ions Self-Reduction Sr3P4O13 Phosphors for Ratiometric Luminescence 
Thermometer. J. Alloys Compd. 2019, 806, 833-840.

(21) Prashanthi, K.; Antić, Ž.; Thakur, G.; Dramićanin, M. D.; Thundat, T. Surface StateInduced Anomalous Negative Thermal Quenching of Multiferroic BiFeO3 Nanowires. Phys. Status Solidi - Rapid Res. Lett. 2018, 12 (1), 1-6.

(22) Prashanthi, K.; Thakur, G.; Thundat, T. Surface Enhanced Strong Visible Photoluminescence from One-Dimensional Multiferroic BiFeO3 Nanostructures. Surf. Sci. 2012, 606 (19-20), L83-L86.

(23) Prashanthi, K.; Gaikwad, R.; Thundat, T. Surface Dominant Photoresponse of Multiferroic BiFeO3 Nanowires under Sub-Bandgap Illumination. Nanotechnology 2013, 24 (50).

(24) Prashanthi, K.; Dhandharia, P.; Miriyala, N.; Gaikwad, R.; Barlage, D.; Thundat, T. Enhanced Photo-Collection in Single BiFeO3 Nanowire Due to Carrier Separation from Radial Surface Field. Nano Energy 2015, 13, 240-248.

(25) Gavrilović, T. V.; Jovanović, D. J.; Lojpur, V.; Dramićanin, M. D. Multifunctional Eu3+and Er3+/Yb3+-Doped GdVO4 Nanoparticles Synthesized by Reverse Micelle Method. Sci. Rep. 2014, 4, 1-9.

(26) Ahadi, K.; Cadien, K. RSC Advances Ultra Low Density of Interfacial Traps with Mixed Thermal and Plasma Enhanced ALD of High- k Gate. RSC Adv. 2016, 6, 16301-16307.

(27) Anti, Ž.; Prashanthi, K.; Jovanovi, D.; Ahadi, K.; Drami, M. D.; Thundat, T. Transparent and Highly Luminescent Dysprosium- Doped GdVO 4 Thin Fi Lms Fabricated by Pulsed Laser Deposition. Thin Solid Films 2017, 638, 332-337.

(28) Ihlefeld, J. F.; Podraza, N. J.; Liu, Z. K.; Rai, R. C.; Xu, X.; Heeg, T.; Chen, Y. B.; Li, J.; Collins, R. W.; Musfeldt, J. L.; et al. Optical Band Gap of BiFeO 3 Grown by MolecularBeam Epitaxy. 2008, 4-6.

(29) Nikolić, M. G.; Antić, Ž.; Ćulubrk, S.; Nedeljković, J. M.; Dramićanin, M. D. Temperature Sensing with Eu3+ Doped TiO2 Nanoparticles. Sensors Actuators B Chem. 2014, 201, 46-50.

(30) Vlaskin, V. A.; Janssen, N.; van Rijssel, J.; Beaulac, R.; Gamelin, D. R. Tunable Dual Emission in Doped Semiconductor Nanocrystals. Nano Lett. 2010, 10 (9), 3670-3674.

(31) Jorge, P.; Martins, M. A.; Trindade, T.; Santos, J. L.; Farahi, F. Optical Fiber Sensing Using Quantum Dots. Sensors 2007, 7 (12), 3489-3534.

(32) Wind, Y.; Saaty, T. L. Marketing Applications of the Analytic Hierarchy Process. 
Manage. Sci. 1980, 26 (7), 641-658.

(33) Saaty, T. L. Decision-Making with the AHP : Why Is the Principal Eigenvector Necessary. 2003, 145, 85-91.

(34) Mohan, K. K.; Prashanthi, K.; Hull, R.; Montemagno, C. D. Risk Assessment of a Multiplexed Carbon Nanotube Network Biosensor. IEEE Sens. J. 2018, 18 (11), $4517-$ 4528 .

(35) Mohan, K. K.; Reformat, M. Z.; Pedrycz, W. Analytic Hierarchy Process and Granularity: Assessment of Risk Severity on Livestock Wellness. In 2012 Annual Meeting of the North American Fuzzy Information Processing Society, NAFIPS 2012; IEEE, 2012.

(36) Mohan, K. K.; Reformat, M. Z.; Pedrycz, W. Assessment of Risk Severity on Livestock Wellness. 2012.

(37) Chang, C.; Wu, C.; Lin, C.; Chen, H. An Application of AHP and Sensitivity Analysis for Selecting the Best Slicing Machine. 2007, 52, 296-307. 


\section{Graphical Abstract}
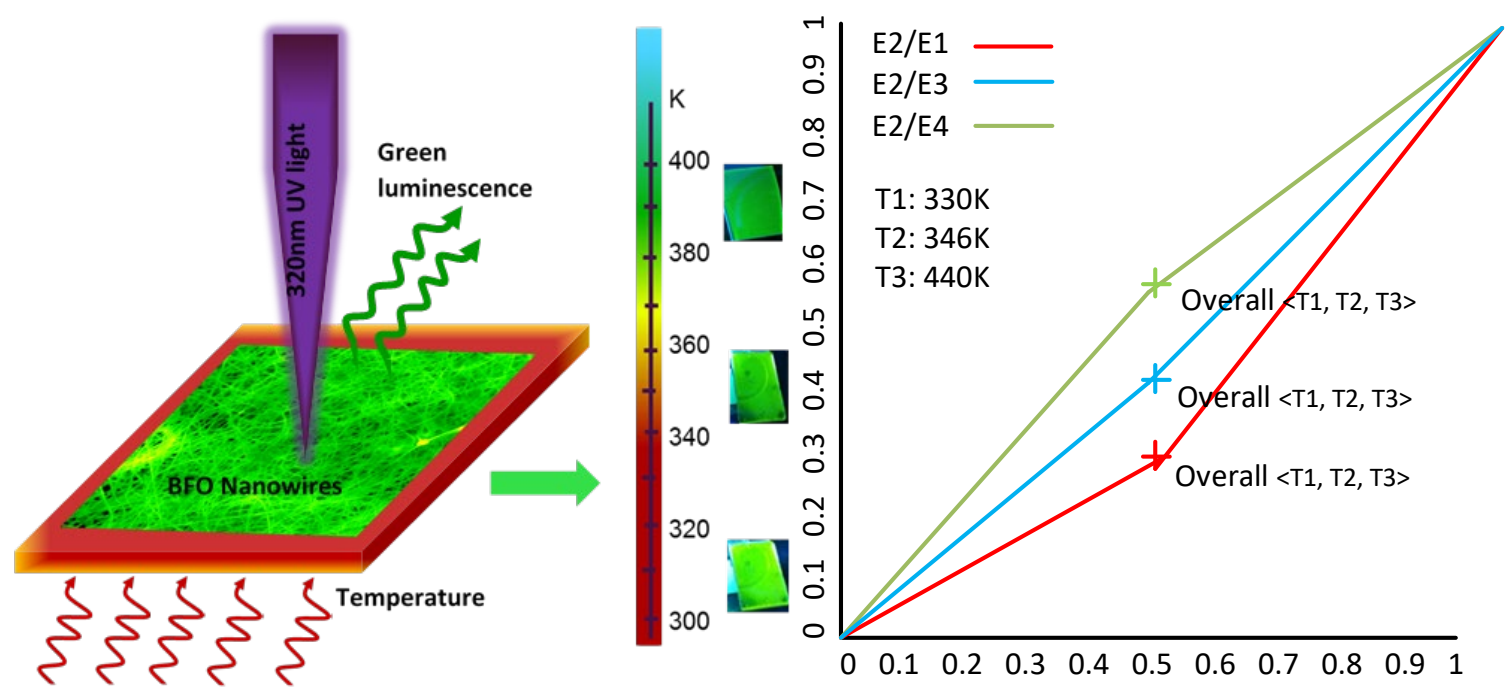

Schematic illustration showing green luminescence of BFO nanowires under UV illumination. With an increasing temperature of nanowires, the intensity of green luminescence increases. Node sensitivities of LIR ratios $(p=0.5)$ at the overall temperature range $(300 \mathrm{~K}-440 \mathrm{~K})$ 